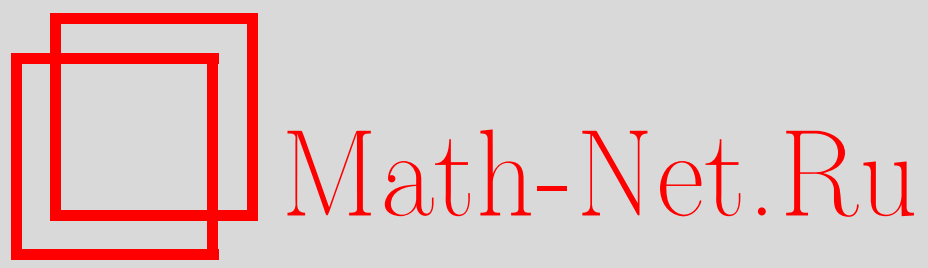

В. С. Рабинович, С. Рох, Фредгольмовость псевдоразностных операторов в весовых пространствах, Функи. анализ и его прил., 2006, том 40, выпуск $1,83-86$

DOI: https://doi.org/10.4213/faa24

Использование Общероссийского математического портала Math-Net.Ru подразумевает, что вы прочитали и согласны с пользовательским соглашением

http: //www . mathnet.ru/rus/agreement

Параметры загрузки:

IP : 52.23 .180 .231

26 апреля 2023 г., 15:43:16

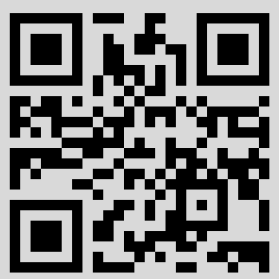




\section{Фредгольмовость псевдоразностных операторов в весовых пространствах ${ }^{*}$}

(c) 2006. В. С. РАБинович, С. Рох

Целью настоящей заметки является изложение некоторых результатов, касающихся фредгольмовости псевдоразностных операторов, действующих в весовых пространствах на $\mathbb{Z}^{N}$ и имеющих следующую форму:

$$
(A u)(x)=(O p(a) u)(x):=\int_{\mathbb{T}^{N}} a(x, t) \hat{u}(t) t^{x} d \mu(t), \quad u \in C_{0}\left(\mathbb{Z}^{N}\right),
$$

где $x=\left(x_{1}, \ldots, x_{N}\right) \in \mathbb{Z}^{N}, \mathbb{T}^{N}:=S^{1} \times \cdots \times S^{1}$ есть $N$-мерный тор, рассматриваемый как мультипликативная группа ( $S^{1}$ - единичная окружность в комплексной плоскости) и $t^{x}=t_{1}^{x_{1}} \cdots t_{N}^{x_{N}}$ для $t \in \mathbb{T}^{N}$ и $x \in \mathbb{Z}^{N}$. Далее,

$$
\mu(t):=\frac{1}{(2 \pi i)^{N}} \frac{d t_{1} \cdots d t_{N}}{t_{1} \cdots t_{N}}
$$

- нормализованная мера Хаара на $\mathbb{T}^{N}$,

$$
\hat{u}(t)=F u(t):=\sum_{x \in \mathbb{Z}^{N}} u(x) t^{-x}, \quad t \in \mathbb{T}^{N},
$$

- дискретное преобразование Фурье функции $u$ и $C_{0}\left(\mathbb{Z}^{N}\right)-$ пространство комплекснозначных функций на $\mathbb{Z}^{N}$ с конечными носителями. Символ $а$ псевдоразностного оператора $O p(a)$ является комплекснозначной функцией, определенной на $\mathbb{Z}^{N} \times \mathbb{T}^{N}$ и такой, что

$$
|a|_{k}:=\sup _{(x, t) \in \mathbb{Z}^{N} \times \mathbb{T}^{N},|\alpha| \leqslant k}\left|\partial_{t}^{\alpha} a(x, t)\right|<\infty
$$

для каждого неотрицательного целого числа $k$. Мы обозначим класс таких функций через $\mathscr{S}$ и будем использовать обозначение $O P \mathscr{S}$ для множества операторов вида (1) с $a \in \mathscr{S}$. Псевдоразностные операторы вида (1) могут рассматриваться как дискретные аналоги стандартных псевдодифференциальных операторов на $\mathbb{R}^{N}$ (см., например, [5]). Отметим, что если существует продолжение символа $a(x, t)$ относительно переменной $x$ на $\mathbb{R}^{N}$, удовлетворяющее оценке $\left|\partial_{x}^{\beta} \partial_{t}^{\alpha} a(x, t)\right| \leqslant C_{\alpha \beta}(1+|x|)^{-|\beta|}$ для любых мультииндексов $\alpha, \beta$, то двойственный относительно дискретного преобразования Фурье оператор $F^{-1} O p(a) F$ яв-

* Доказательства приведенных в заметке результатов опубликованы в Acta Applicandae Math., 84, 55-96 (2004). 
ляется псевдодифференциальным оператором на торе $\mathbb{T}^{N}$. (См., например, [1] и указанную там литературу.)

Если $a$ есть полином относительно $t \in \mathbb{T}^{N}$, то $O p(a)$ является разностным оператором на $\mathbb{Z}^{N}$. Отметим, что наиболее важными и лучше всего изученными разностными операторами являются операторы порядка два в размерности один, которые также называются операторами Якоби. Имеется обширная библиография, посвященная этим операторам (см., например, монографии [3, 4, 6] и приведенную там литературу).

Пусть $X$ - банахово пространство и $L(X)$ - банахова алгебра всех ограниченных линейных операторов, действующих в $X$. Далее, для $p \geqslant 1$ рассмотрим банахово пространство $l^{p}\left(\mathbb{Z}^{N}\right)$ всех комплекснозначных функций $f$ на $\mathbb{Z}^{N}$, таких, что $\|f\|_{l^{p}\left(\mathbb{Z}^{N}\right)}:=\left(\sum_{x \in \mathbb{Z}^{N}}|f(x)|^{p}\right)^{1 / p}<\infty$, если $p<\infty$, и $\|f\|_{l^{\infty}\left(\mathbb{Z}^{N}\right)}:=$ $\sup _{x \in \mathbb{Z}^{N}}|f(x)|<\infty$, если $p=\infty$. Наконец, для заданной положительной функции $w$ на $\mathbb{Z}^{N}$ будем обозначать через $l_{w}^{p}\left(\mathbb{Z}^{N}\right)$ весовое пространство функций $u$ на $\mathbb{Z}^{N}$ с нормой $\|u\|_{l_{w}^{p}\left(\mathbb{Z}^{N}\right)}:=\|w u\|_{l^{p}\left(\mathbb{Z}^{N}\right)}<\infty$.

TЕОРемА 1. Псевдоразностный оператор $О р(a) \in O P \mathscr{S}$ ограничен в $l^{p}\left(\mathbb{Z}^{N}\right)$ для любого $p, 1 \leqslant p \leqslant \infty, u\|A\|_{L\left(l^{p}\left(\mathbb{Z}^{N}\right)\right)} \leqslant C|a|_{2 k}$, где $2 k>N$, а константа $C>0$ не зависит от $A$.

Для каждого $p \in[1, \infty]$ псевдоразностные операторы из $O P \mathscr{S}$ образуют некоторую (незамкнутую) подалгебру в $L\left(l^{p}\left(\mathbb{Z}^{N}\right)\right)$ в том смысле, что сумма и произведение операторов из $O P \mathscr{S}$ снова принадлежат $O P \mathscr{S}$. Более того, если $A \in O P \mathscr{S}$ и $A$ действует на $l^{p}\left(\mathbb{Z}^{N}\right)$ с $p<\infty$, то и $A^{*} \in O P \mathscr{S}$. Формулы для символов произведения и сопряженного оператора подобны соответствующим, хорошо известным формулам для псевдодифференциальных операторов на $\mathbb{R}^{N}$ (см., например, [5, Chap. 2]).

Для $r>1$ положим $\mathbb{K}_{r}=\{z \in \mathbb{C}: 1 / r<|z|<r\}$ и для каждого набора $r=$ $\left(r_{1}, \ldots, r_{N}\right)$ из $N$ вещественных чисел $r_{j}>1$ рассмотрим $N$-мерную кольцевую область $\mathbb{K}_{r}^{N}=\mathbb{K}_{r_{1}} \times \cdots \times \mathbb{K}_{r_{N}}$ в $\mathbb{C}^{N}$.

Пусть $\mathscr{S}\left(\mathbb{K}_{r}^{N}\right)$ обозначает множество всех функций $a: \mathbb{Z}^{N} \times \mathbb{K}_{r}^{N} \rightarrow \mathbb{C}$, которые являются аналитическими по переменной $t \in \mathbb{K}_{r}^{N}$ и удовлетворяют оценкам $|a|_{k}=\sum_{|\alpha| \leqslant k} \sup _{x, t \in \mathbb{Z}^{N} \times \mathbb{K}_{r}^{N}}\left|\partial_{t}^{\alpha} a(x, t)\right|<\infty$ для каждого неотрицательного целого числа $k$. Каждой функции $a \in \mathscr{S}\left(\mathbb{K}_{r}^{N}\right)$ сопоставим псевдоразностный оператор $O p(a)$ на $C_{0}\left(\mathbb{Z}^{N}\right)$. Обозначим класс всех таких псевдоразностных операторов с символами из $\mathscr{S}\left(\mathbb{K}_{r}^{N}\right)$ через $О Р \mathscr{S}\left(\mathbb{K}_{r}^{N}\right)$.

ОПРЕДЕЛЕНИЕ 2. Пусть $W\left(\mathbb{K}_{r}^{N}\right)$ обозначает класс всех экспоненциальных весов вида $w=e^{v}$, где $v$ есть ограничение на $\mathbb{Z}^{N}$ вещественной функции $\tilde{v} \in$ $C^{1}\left(\mathbb{R}^{N}\right)$, такой, что $\left|\partial \tilde{v}(x) / \partial x_{j}\right|<\log r_{j}$ для каждого $x \in \mathbb{R}^{N}$ и любого $j \in$ $\{1, \ldots, N\}$.

Сформулированное ниже предложение сводит изучение псевдоразностных операторов, принадлежащих $O P \mathscr{S}\left(\mathbb{K}_{r}^{N}\right)$ и действующих в $l_{w}^{p}\left(\mathbb{Z}^{N}\right)$, к исследованию псевдоразностных операторов, принадлежащих $O P \mathscr{S}$ и действующих в $l^{p}\left(\mathbb{Z}^{N}\right)$.

ПРЕДЛОЖЕНИЕ 3. Если $a \in \mathscr{S}\left(\mathbb{K}_{r}^{N}\right)$ u $w \in W\left(\mathbb{K}_{r}^{N}\right)$, то весовой оператор $w O p(a) w^{-1}$ (первоначально определенный на $\left.C_{0}\left(\mathbb{Z}^{N}\right)\right)$ принадлежст ОРS,, следовательно, Ор $(a)$ есть ограниченный оператор в $l_{w}^{p}\left(\mathbb{Z}^{N}\right)$ для кажсдого $p \in$ $[1, \infty]$. 
Будем говорить, что функция $u \in l^{\infty}\left(\mathbb{Z}^{N}\right)$ является медленно осциллирующей, если

$$
\lim _{x \rightarrow \infty}(u(x+y)-u(x))=0
$$

для каждого $y \in \mathbb{Z}^{N}$, и будем говорить, что вес $w=e^{v}$ является медленно осциллирующим, если ограничение $\partial \tilde{v} / \partial x_{j}$ на $\mathbb{Z}^{N}$ является медленно осциллирующей функцией для каждого $j$.

Символ $a \in \mathscr{S}\left(\mathbb{K}_{r}^{N}\right)$ называется медленно осциллирующим, если

$$
\lim _{x \rightarrow \infty} \sup _{t \in \mathbb{K}_{r}^{N}}|a(x+y, t)-a(x, t)|=0
$$

для каждой точки $y \in \mathbb{Z}^{N}$. (Отметим, что псевдодифференциальные операторы на $\mathbb{R}^{N}$ с медленно осциллирующими символами рассматривались впервые в [2].)

Будем говорить, что $\lambda \in \mathbb{C}$ принадлежит существенному спектру оператора $A$, если оператор $A-\lambda I$ не является фредгольмовым на $X$. Будем обозначать существенный спектр через $\operatorname{sp}_{X}^{\mathrm{ess}}(A)$, а через $\operatorname{sp}_{X}(A)$ обозначим обычный спектр оператора $A$ на $X$.

ТЕОРемА 4. Пусть символ $a \in \mathscr{S}\left(\mathbb{K}_{r}^{N}\right)$ и вес $w \in W\left(\mathbb{K}_{r}^{N}\right)$ являются медленно осииллирующими. Тогда $О p(a): l_{w}^{p}\left(\mathbb{Z}^{N}\right) \rightarrow l_{w}^{p}\left(\mathbb{Z}^{N}\right), p \in[1, \infty]$, является оператором Фредгольма в том и только том случае, когда

$$
\lim _{R \rightarrow \infty} \inf _{|x| \geqslant R, t \in \mathbb{T}^{N}}\left|a\left(x, e^{\partial_{x_{1}} v(x)} t_{1}, \ldots, e^{\partial_{x_{N}} v(x)} t_{N}\right)\right|>0 .
$$

Более того,

¿əe

$$
\operatorname{sp}_{l_{w}^{p}\left(\mathbb{Z}^{N}\right)}^{\mathrm{ess}}(O p(a))=\bigcup_{h}\left\{\lambda \in \mathbb{C}: \lambda=\tilde{a}_{h}(t), t \in \mathbb{T}^{N}\right\}
$$

$$
\tilde{a}_{h}(t)=\lim _{n \rightarrow \infty} a\left(h(n), e^{\partial_{x_{1}} v(h(n))} t_{1}, \ldots, e^{\partial_{x_{N}} v(h(n))} t_{N}\right)
$$

и где объединение в (2) берется по всем последовательностям $h(n)$, для которых предел в (3) существует.

ТЕОрема 5 (принцип Фрагмена-Линделёфа). Пусть а и $w$ такие же, как в предьдущей теореме, $u \lim _{x \rightarrow \infty} w(x)=+\infty$. Пусть

$$
\lim _{R \rightarrow \infty} \inf _{|x| \geqslant R, t \in \mathbb{K}_{r}^{N}}|a(x, t)|>0 .
$$

Если $p \in(1, \infty)$ и и есть решение уравнения $O p(a) u=f c f \in l_{w}^{p}\left(\mathbb{Z}^{N}\right)$, которое первоначально принадлежит $l_{w^{-1}}^{p}\left(\mathbb{Z}^{N}\right)$, mо $u \in l_{w}^{p}\left(\mathbb{Z}^{N}\right)$.

Применим сформулированные выше результаты к исследованию дискретных операторов Шрёдингера вида

$$
(\mathscr{H} u)(x)=\sum_{j=1}^{N}\left(V_{e_{j}}+V_{-e_{j}}\right) u(x)+a(x) u(x), \quad x \in \mathbb{Z}^{N},
$$

где $e_{j}=\left(0, \ldots, 1_{j}, \ldots, 0\right)$ - единичный вектор, $V_{-h} u(x)=u(x-h), h \in \mathbb{Z}^{N}-$ оператор сдвига, потенциал $a$ принадлежит $l^{\infty}\left(\mathbb{Z}^{N}\right)$, и $а$ есть вещественная медленно осциллирующая функция. Сформулированный ниже результат есть следствие теоремы 4. 
Tеорема 6. Существенный спектр оператора $\mathscr{H}$ не зависит от $p \in[1, \infty]$ $u \operatorname{sp}_{l p\left(\mathbb{Z}^{N}\right)}^{\mathrm{ess}} \mathscr{H}=\left[-2 N+a_{-}, 2 N+a_{+}\right]$, где $a_{-}:=\liminf _{x \rightarrow \infty} a(x) u a_{+}:=\limsup _{x \rightarrow \infty} a(x)$.

Для применения принципа Фрагмена-Линделёфа (теорема 3) к оператору Шрёдингера $\mathscr{H}$ мы должны рассмотреть два взаимно исключающих случая: $(-) a_{+}<-2 N$ и $(+) a_{-}>2 N$.

Теорема 7. Пусть $w=e^{v}$, где $\lim _{x \rightarrow \infty} v(x)=+\infty$. Предположим, что в случае (-)

$$
\left|\frac{\partial v(x)}{\partial x_{j}}\right| \leqslant \log \left(\frac{\left|a_{+}\right|-\varepsilon}{2 N}+\sqrt{\left(\frac{\left|a_{+}\right|-\varepsilon}{2 N}\right)^{2}-1}\right), \quad j=1, \ldots, N,
$$

а в случае $(+)$

$$
\left|\frac{\partial v(x)}{\partial x_{j}}\right| \leqslant \log \left(\frac{\left|a_{-}\right|-\varepsilon}{2 N}+\sqrt{\left(\frac{\left|a_{-}\right|-\varepsilon}{2 N}\right)^{2}-1}\right), \quad j=1, \ldots, N,
$$

для достаточно больиих $x \in \mathbb{R}^{N}$ и достаточно мальх $\varepsilon>0$. Пусть и есть решение уравнения $\mathscr{H} u=f c f \in l_{w}^{2}\left(\mathbb{Z}^{N}\right)$, которое первоначально принадлежст пространству $l_{w^{-1}}^{2}\left(\mathbb{Z}^{N}\right)$. Тогда $u \in l_{w}^{2}\left(\mathbb{Z}^{N}\right)$.

Из этой теоремы получается оценка поведения собственных функций дискретного оператора Шрёдингера на бесконечности.

\section{ЛитерАТУРА}

1. Агранович M. C. В кн.: Итоги науки и техники. Современные проблемы математики. Фундаментальные направления. Т. 63,1990, с. 5-130. 2. Грушин В. В. Функц. анализ и его прил., 4, No. 3, 37-50 (1970). 3. Deift P. Orthogonal Polynomials and Random Matrices: A Riemann-Hilbert Approach, Courant Lect. Notes in Math., Vol. 3, Amer. Math. Soc., Providence, R.I., 2000. 4. Jirari A. Memoirs Amer. Math. Soc., Vol. 542, Providence, R.I., 1995. 5. Taylor M. E. Pseudodifferential Operators. Princeton University Press, Princeton, New Jersey, 1981. 6. Teschl G. Jacobi Operators and Completely Integrable Nonlinear Lattices. Math. Surveys and Monographs, Vol. 72, Amer. Math. Soc., Providence, R.I., 1999.

Instituto Politécnico Nacional, ESIME-Zacatenco, Mexico e-mail: vladimir_rabinovich@hotmail.com

Поступило в редакцию Technische Universität Darmstadt, Fachbereich Mathematik e-mail: roch@mathematik.tu-darmstadt.de 14 апреля 2004 г. 